\title{
Tuberculosis Osteoarticular Primaria: Reaparición de una Patología Olvidada
}

\section{Primary osteoarticular tuberculosis: the reappearance of a forgotten pathology}

\author{
Enrique Vergara-Amador ${ }^{1}$, Fernando Galván-Villamarin² y \\ Marcela Piña-Quintero ${ }^{1}$
}

\author{
1 Departamento de Cirugía, Facultad de Medicina, Universidad Nacional de Colombia. \\ emvergaraa@unal.edu.co; enriquevergaraa@yahoo.com \\ 2 Hospital de la Misericordia. Bogotá, Colombia \\ Recibido 6 Octubre 2007/Enviado para Modificación 26 Mayo 2007/Aceptado 5 Julio 2007
}

\section{RESUMEN}

Presentamos dos casos de infección tuberculosa osteoarticular primaria, uno en el astrágalo y el otro en la columna vertebral, en pacientes aparentemente sanos y sin antecedentes epidemiológicos. Son casos representativos de la tuberculosis osteoarticular primaria, mostrando un aumento gradual en la última década en nuestro país, en pacientes sin alteraciones inmunológicas y sin antecedentes familiares o de contacto..

Palabras Clave: Tuberculosis osteoarticular, tuberculosis ósea, astrágalo (fuente: DeCS, BIREME).

\section{ABSTRACT}

2 cases of osteoarticular tuberculosis infection are presented (one in the talus and the other in the spine) in seemingly healthy patients having no epidemic antecedents. These are representative cases of primary osteoarticular tuberculosis, showing a gradual increase over the last decade in Colombia in patients without immunological alterations and having no family antecedents or history of contact.

Keys Words: Osteoarticular tuberculosis, bone tuberculosis, talus (source: $\mathrm{MeSH}$, $N L M)$.

L a tuberculosis del sistema osteoarticular a nivel mundial se ha considerado que corresponde al 3\% de las tuberculosis extrapulmonares, como cadera y rodilla. El diagnóstico es generalmente tardío debido a su baja prevalencia, a la habilidad de esta entidad de mimificar otras 
enfermedades y a la falta de sospecha por parte del médico (1). En el panorama colombiano se encuentran reportes de compromiso osteoarticular entre el 2 al 5, similar a la reportada en la literatura, con mayor incidencia en la columna vertebral. (2) El propósito de este reporte es el de presentar dos casos de tuberculosis osteoarticular en niños aparentemente sanos, sin ningún antecedente de tuberculosis pulmonar o en la familia, el primero con compromiso del Astrágalo, sitio exótico, y el segundo con compromiso vertebral.

\section{Caso 1}

Niña de 17 meses, con cuadro clínico de Marzo del 2005, de 3 meses de evolución con dolor y edema en tobillo derecho sin antecedente de trauma. Tratada inicialmente como una sinovitis transitoria con analgésico sin mejoría. Cuadro hemático con leucocitosis y neutrofilia, PCR elevada. La radiografía de pie mostró una zona osteolítica en el cuerpo del astrágalo. (Figura 1). Se realizó artrocentesis que fue negativa para proceso infeccioso. Resonancia magnética a los 45 días del inicio del cuadro mostró alteración de la arquitectura del astrágalo con abundante líquido tibio talar.

Se remite a nuestra institución encontrándose en buen estado general, edema y limitación de la movilidad del tobillo derecho. Presentaba leucocitosis, VSG y PCR elevada y un hemocultivo positivo para staphilococo capitis. Rx mostró lesión osteolítica del astrágalo con compromiso de la superficie articular. Con impresión diagnóstica de artritis séptica se llevo a cirugía para drenaje donde se obtuvo líquido blanquecino espeso sugestivo de proceso infeccioso. Se inició tratamiento con oxacilina y amikacina. El resultado del cultivo de secreción fue negativo y la patología reportó material óseo con cambios granulomatosos con necrosis caseificante y evidencia de dos estructuras bacilo alcohol resistente correspondiente a tuberculosis.

La investigación epidemiológica para tuberculosis fue negativa. El jugo gástrico fue negativo para bacilo de Koch. Radiografía de tórax normal y prueba de tuberculina de $8 \mathrm{~mm}$., considerada normal. Con el diagnóstico de infección tuberculosa del astrágalo se inicio tratamiento triconjugado con isoniacida, etambutol y rifampicina. La respuesta clínica fue satisfactoria.

\section{Caso 2}

Niña de 15 meses de edad con cuadro de 4 meses de evolución de irritabilidad, fiebre intermitente y pérdida de peso progresivas, tratada como infección del tracto respiratorio sin mejoría. Al mes de iniciado el cuadro la niña presento dolor dorso lumbar que se interpreto como infección de vías urinarias. 
$\mathrm{Al}$ examen físico buenas condiciones generales, irritable e hiperreflexia en miembros inferiores. Cuadro hemático normal, VSG, PCR y hemocultivos negativos. Prueba de tuberculina negativa y sin antecedentes epidemiológicos de tuberculosis La resonancia magnética de la columna evidencio cambios sugestivos de espondilodiscitis T12- L1 con absceso peridural compresivo del canal y absceso de psoas izquierdo, hallazgos sugestivos de tuberculosis, por lo cual se inició tratamiento triconjugado con pirazinamida, isoniacida y rifampicina. A los 15 días de tratamiento, la resonancia de control mostró persistencia de la colección epidural con compresión del cono medular a nivel T12-L1 (Figura 2). Se llevó a cirugía para decomprensión y fijación. La biopsia reportó granulomas caseificantes tanto en tejido cartilaginoso como óseo, compatible con tuberculosis. La paciente es dada de alta a las 6 semanas con esquema triconjugado para TBC.

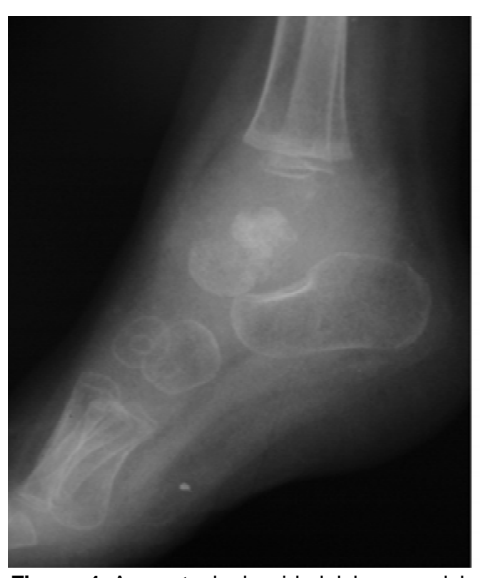

Figura 1. Aumento de densidad del cuerpo del astrágalo con zonas osteolìticas y aparente fractura sobre el cuello del astrágalo.

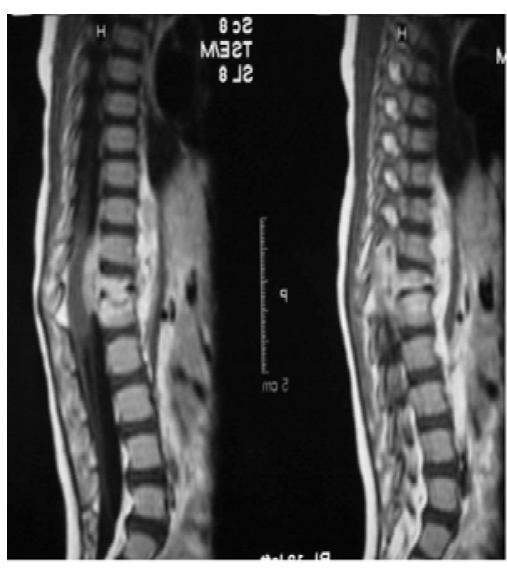

Figura 2. Compromiso de las vértebras $\mathrm{T} 12$ y L1 con colapso y absceso epidural que compromete el canal medular, además levantamiento de todo el ligamento longitudinal anterior.

\section{DISCUSION}

La tuberculosis se mantiene como un problema de salud pública internacional. Actualmente, se estima que un tercio de la población sufre de tuberculosis en el mundo $(3,4)$. La incidencia anual es de 8 millones, con una tasa de mortalidad de 3 millones por año $(5,6)$. El aumento de la incidencia está directamente relacionado con el aumento en el número de pacientes inmunosuprimidos (pacientes VIH positivos), migración, desarrollo de micobacterias resistentes al tratamiento antituberculoso, malnutrición, bajo nivel socioeconómico, edad y aumento del número de trabajadores de salud expuestos a la enfermedad (1- 
6). En Colombia, se ha observado una tendencia al aumento de la tuberculosis, dada no sólo por los anteriores factores, sino también por los cambios a nivel de las políticas de salud, con respecto al programa de Tuberculosis, con una reducción de los recursos, afectando las labores de localización de casos, de supervisión y de capacitación (3).

La tuberculosis osteoarticular representa el 1 al $3 \%$ de las formas extrapulmonares donde la columna vertebral ocupa alrededor del $50 \%(1,6,7)$. En el $10 \%$ se pueden encontrar lesiones múltiples. Existen diferentes formas de diseminación del microorganismo hacia el sistema osteoarticular, predominando la hematógena, secundaria de un foco primario activo o latente ya sea en pulmón, ganglios o vísceras, que llega al sistema músculo esquelético por canales vasculares arteriales (8). En la presentación articular la forma de diseminación es por vía directa a través de los vasos subsinoviales o por vía indirecta a través del hueso adyacente. El curso es lento, produciendo hipertrofia y formación de tejido de granulación en la sinovial con derrame articular y por ultimo erosión del hueso (1).

El diagnóstico de tuberculosis se basa en 7 criterios: clínico, epidemiológico, prueba cutánea de la tuberculina (PPD), radiológico, baciloscopia, cultivo e histopatológico (2). Sin embargo puede presentarse dificultad para hacer el diagnóstico de esta patología, ya que en muchos casos puede comportarse como osteomielitis aguda o subaguda o como una artritis séptica $(5,6,9,10)$. La presentación clínica es de inicio insidioso. La prueba de tuberculina (PPD) generalmente es positiva, teniendo en cuenta que en pacientes con anergia por inmunosupresión o por desnutrición puede dar resultado falso negativo $(4,6)$.

Los cambios radiológicos aparecen después de la cuarta semana. Inicialmente se observa reacción perióstica y osteopenia. Más tarde aparecen zonas líticas, quistes subcondrales y erosiones $(1,5,6)$. Phemister enunció la triada radiológica conformada por osteoporosis yuxtaarticular, erosiones marginales y disminución del espacio articular $(4,9)$.

La tomografía axial nos ayuda a demostrar la fase destructiva de la enfermedad con compromiso del hueso (5). Por otra parte, la resonancia magnética tiene mayor valor en el diagnóstico y en el pronóstico, ayuda a detectar lesiones en su fase temprana, derrames articulares, presencia de cuerpos libres calcificados, engrosamientos de la cápsula y la sinovial, compromiso neurovascular y compromiso de tejidos blandos. 
Todos los anteriores métodos hacen una aproximación al diagnóstico. La confirmación se realiza con la biopsia ya sea por aguja guiada o por cirugía abierta. Se observa tejido granulomatoso con necrosis de caseificación. La presencia del bacilo al microscopio, sólo se encuentra positiva en menos del 20 a $30 \%$ de los casos, debido a que es paucibacilar $(5,611)$, lo que nos haría tener más presente los otros criterios diagnósticos para iniciar tratamiento.

El tratamiento de esta entidad en el compromiso osteoarticular generalmente es médico con esquemas de 12 a 18 meses $(1,6,8)$. El tratamiento quirúrgico no se recomienda en las fases iniciales a menos que el paciente requiera curetaje y desbridamiento por infecciones concomitantes $(1,6)$ o en el caso de compromiso de la columna vertebral cuando afecta la estabilidad. El pronóstico dependerá de terapia médica instaurada tempranamente, del hueso o de la articulación comprometida $(7,12)$.

Pocos reportes existen en nuestro medio respecto a las formas osteoarticulares (2,3). Arciniegas y Orjuela (3) reportan 102 casos extrapulmonares, con solo 5 de ellos osteoarticulares, 3 en la rodilla y 2 en la columna. En la única serie encontrada en Colombia de tuberculosis osteoarticular, realizada por el Hospital San Vicente de Paul en Medellín, encontraron 47 casos entre 1994 y 2004, que correspondía al 3,2 \% de todas las formas de tuberculosis encontradas. La forma espinal ocupó el 74,5 \% de todas y el tobillo solo 3 casos (2), muy similar a la encontrada en la literatura mundial.

Llama la atención de los casos mostrados por nosotros, la presentación de la enfermedad en pacientes aparentemente sanos, de un entorno social y cultural bueno y sin antecedentes epidemiológicos de tuberculosis. No encontramos un reporte sobre la presencia de la enfermedad en un grupo con estas características, por lo tanto es importante que la comunidad médica esté atenta para la detección temprana y tratamiento oportuno en estos pacientes en donde la sospecha inicial es mínima.

Por último en nuestro país, con los cambios de políticas en salud, aumento de la incidencia de VIH, de los índices de pobreza y el desplazamiento forzado, se crea la necesidad de nuevas políticas, tanto para la detección, tratamiento y seguimiento de los pacientes como en la organización de un sistema de referencia adecuado para lograr un impacto en el control de esta patología $(2,13)$

Agradecimientos. Al Dr. Leonardo Duplat por el aporte del caso clínico $\mathrm{N}^{\circ}$ dos. 


\section{REFERENCIAS}

1. Tuli S. General Principles of Osteoarticular Tuberculosis Clinical Orthop Relat Res. May 2002; (398): 11-19.

2. López M, Barros E, Uribe A, Toro A, López J. Perfiles epidemiológico y clínico de la tuberculosis osteoarticular, estudio observacional en el Hospital Universitario San Vicente de Paul de Medellín 1994-2004. Iatreia, Sept 2003; 18:279-288.

3. Arciniegas W, Orjuela D. Tuberculosis extrapulmonar revisión de 102 casos en el Hospital Universitario San Jorge de Pereira 2000-2004. Facultad Ciencias de la Salud Universidad Tecnológica de Pereira.

4. Cooper DG, Fazal MA, William RL. Isolated tuberculous osteomyelitis of the bones of the hindfoot: a case report and review of the literature. Foot and ankle Surg. 2001; (7): 181-185.

5. Vohra R, Kang HS, Dogra S, Sharma R. Tuberculous Osteomyelitis. J Bone and Joint Surg. 1997 Jul; 79-B(4): 562-566.

6. Watts H, Lifeso R. Tuberculosis of Bones and Joints. J Bone and Joint Surg. Feb 1996; 78-A(2), 288-299.

7. Dhillon M, Nagi O. Tuberculosis of the Foot and Ankle. Clin Orthop Related Research. May 2002; (398):107-113.

8. Stuart D. Local osteo-articular tuberculosis complicating closed fractures. Report of two cases. J Bone and Joint Surg. May 1976; (58-B):248-249.

9. Mittal R, Gupta V, Rastogi S. Tuberculosis of the foot. J Bone and Joint Surg. Nov 1999; 81-B(6): 997-1000.

10. Babhulkar S, Pande S. Unusual manifestations of Osteoarticular Tuberculosis. Clin Orthop Relat Res. May 2002; (398): 114-120.

11. Teklali Y, Fellous Z. Tuberculosis of the talus in the child. Eur J Orthop Surg Traumatol. 2003; (13): 52-54.

12. Anand A, Sood LK. Isolated tuberculosis of talus without ankle and subtalar joint involvement. Med J Malaysia. Sep 2002; (57): 371-373.

13. Díaz ML, Muñoz S, García LB. Tuberculosis en el Hospital Universitario San José, Popayán, 1998-2000. Biomédica 2004;24 (Suppl.1):92-101. 